\title{
ON THE CASES OF KIRCHHOFF AND CHAPLYGIN OF THE KIRCHHOFF EQUATIONS
}

\author{
VLADIMIR DRAGOVIĆC ${ }^{1,2}$, BORISLAV GAJIĆ ${ }^{1}$
}

\begin{abstract}
It is proven that the general Kirchhoff case of the Kirchhoff equations for $B \neq 0$ is not algebraic complete integrable system. Similar analytic behavior of the general solution of the Chaplygin case is detected. Four-dimensional analogues of the Kirchhoff and the Chaplygin cases are defined on $e(4)$ with the standard Lie-Poisson bracket.
\end{abstract}

\section{INTRODUCTION}

Starting from the fact that both in the Euler and the Lagrange cases of motion of a heavy rigid body fixed at a point, the general solutions are meromorphic as functions of complex time, Sofia Kowalevski in [11 had proposed a problem of finding all cases of such rigid-body motion that have general solutions with the same analytic properties. Kowalevski had discovered that there was only one extra case, with such dynamics, what is nowadays called the Kowalevski case. Moreover, Kowalevski had found an additional polynomial first integral (of fourth degree) and she solved the system in terms of genus two theta-functions. In mid 1970's Kozlov proved (see[12]) a nonexistence of fourth analytical first integral in heavy rigid-body dynamics, except in the three cases mentioned above. Thus, it appears that in the case of motion of a heavy rigid body fixed at a point, a fourth first integral exists exactly in cases when the general solutions are meromorphic functions of complex time.

Further development of such analytic theory of differential equations was associated with Painlevé.

A modern theory of so-called algebraic complete integrable systems has been performed by many authors, and the most fundamental results and precise definitions can be found in the book [2] (see also [1]).

It has generally been assumed among the experts as a strong believe that the same parallelism between complete integrability and meromorphicity of general solutions exists also in a similar case of motion of a rigid body in an ideal incompressible fluid which rest in infinity, described by so-called Kirchhoff equations. The known integrable cases are those of Kirchhoff, the Clebsch case, the Steklov-Lyapunov case, the Sokolov case. For the full list of known integrable cases see for example [3].

MCS: 70E40, 70E45, 70E20 
The main result of the present paper is the proof that the general Kirchhoff case, for $B \neq 0$ (see below for notations) is not algebraic complete integrable. Its general solution is not a meromorphic function of complex time. Thus, there are classical, very well-known cases of integrable dynamics of Kirchhoff equations which are not algebraic integrable and which do not pass the so-called Kowalevski-Painlevé test. In the proof we use the method of small parameter, and historically it goes back to Poincaré.

We also study the Chaplygin case, which is classically known perturbation of the Kirchhoff case and known to be non-integrable (see [13]). By the same method of the small parameter we detect similar behavior of the general solution of the Chaplygin case as in the general Kirchhoff case.

Finally, we study four-dimensional generalizations and we define appropriate analogues of the Kirchhoff and of the Chaplygin case.

\section{Three-Dimensional Kirchhoff equations}

The motion of a rigid body in an ideal incompressible fluid which rest in infinity is described by the Kirchhoff equations [10, 14, 3]:

$$
\begin{aligned}
\dot{M} & =M \times \frac{\partial H}{\partial M}+p \times \frac{\partial H}{\partial p}, \quad \dot{p}=p \times \frac{\partial H}{\partial M} \\
H & =\frac{1}{2}\langle A M, M\rangle+\langle B M, p\rangle+\frac{1}{2}\langle C p, p\rangle
\end{aligned}
$$

Here $M$ and $p$ are the impulsive moment and the impulsive force. The matrices $B$ and $C$ are symmetric, $A$ is a symmetric positive-definite matrix, and they reflect the geometry and the mass distribution of the body. The equations (11) are Hamiltonian on Lie algebra $e(3)$ with the standard Poisson structure

$$
\left\{M_{i}, M_{j}\right\}=-\epsilon_{i j k} M_{k}, \quad\left\{M_{i}, p_{j}\right\}=-\epsilon_{i j k} p_{k}
$$

The Casimir functions are $F_{2}=\langle M, p\rangle, \quad F_{3}=\langle p, p\rangle$. Thus, for complete integrability in the Liouville sense, apart from the Hamiltonian $F_{1}=H$, one needs one additional first integral. The Kirchhoff case (1870) is defined by the conditions (see [10]):

$$
A=\operatorname{diag}\left(a_{1}, a_{1}, a_{3}\right), B=\operatorname{diag}\left(b_{1}, b_{1}, b_{3}\right) C=\operatorname{diag}\left(c_{1}, c_{1}, c_{3}\right) .
$$

An additional first integral is $F_{4}=M_{3}$. Thus, the Kirchhoff case is in a sense, analogous to the Lagrange case of motion of a heavy rigid body fixed at a point.

\section{Algebraic integrability of Kirchhoff CASe}

When $B=0$, the Kirchhoff case can be seen as a special case of the Clebsch integrable case defined by $\frac{c_{2}-c_{3}}{a_{1}}+\frac{c_{3}-c_{1}}{a_{2}}+\frac{c_{1}-c_{2}}{a_{3}}=0$. A Lax representation and a higher-dimensional generalization of the Clebsch case are given by Perelomov (see [16]). It is known that the Clebsch case is algebraic complete integrable system (see for example [15]). 
Let us pass to the case when $B \neq 0$. The Kowalevski-Painlevé test gives necessary conditions for an $n$-dimensional system to be algebraic completely integrable: the system has to posses Laurent solutions depending of $n-1$ parameters. The vector field in the Kirchhoff equations is homogeneous quadratic in $M_{i}, p_{i}$. One can expect solutions which admit poles of the first order: $M_{i}=\frac{M_{i}^{(0)}}{t}+M_{i}^{(1)}+\ldots, p_{i}=\frac{p_{i}^{(0)}}{t}+p_{i}^{(1)}+\ldots$ By plugging it into equations (1) one gets for $M_{i}^{(0)}$ and $p_{i}^{(0)}$ :

$$
\begin{aligned}
-M_{1}^{(0)} & =\left(b_{3}-b_{1}\right) M_{2}^{(0)} p_{3}^{(0)}+\left(c_{3}-c_{1}\right) p_{2}^{(0)} p_{3}^{(0)} \\
-M_{2}^{(0)} & =\left(b_{1}-b_{3}\right) M_{1}^{(0)} p_{3}^{(0)}+\left(c_{1}-c_{3}\right) p_{1}^{(0)} p_{3}^{(0)} \\
-M_{3}^{(0)} & =0 \\
-p_{1}^{(0)} & =-a_{1} M_{2}^{(0)} p_{3}^{(0)}+\left(b_{3}-b_{1}\right) p_{2}^{(0)} p_{3}^{(0)} \\
-p_{2}^{(0)} & =a_{1} M_{1}^{(0)} p_{3}^{(0)}+\left(b_{1}-b_{3}\right) p_{1}^{(0)} p_{3}^{(0)} \\
-p_{3}^{(0)} & =a_{1}\left(p_{1}^{(0)} M_{2}^{(0)}-p_{2}^{(0)} M_{1}^{(0)}\right)
\end{aligned}
$$

From the first integrals one can conclude that $M_{1}^{(0)} p_{1}^{(0)}+M_{2}^{(0)} p_{2}^{(0)}+M_{3}^{(0)} p_{3}^{(0)}=$ 0, but from (2) one has: $M_{1}^{(0)} p_{1}^{(0)}+M_{2}^{(0)} p_{2}^{(0)}+M_{3}^{(0)} p_{3}^{(0)}=\left(b_{3}-b_{1}\right) p_{3}^{(0)^{2}}$. Consequently, we have that $b_{3}=b_{1}$ or $p_{3}^{(0)}=0$. In the first case one has that the mixed part $\langle B M, p\rangle$ in the Hamiltonian is a Casimir function. Thus, this part can be dropped from the Hamiltonian and we come to the case $B=0$. If $p_{3}^{(0)}=0$, equations (2) have only a trivial solution. Thus, they do not admit a Laurent solution with poles of the first order.

Thus, we have the following

Lemma 1. a) In the case $B=0$ the Kirchhoff case as a special case of the Clebsch case is algebraic complete integrable system.

b) In the case $B \neq 0$ the Kirchhoff case does not pass the KowalevskiPainlevé test.

Thus, a natural question arises:

In the general case $B \neq 0$ is the Kirchhoff case algebraic complete integrable system?

In order to answer the question, we are going to apply the method of small parameter, following some ideas of Lyapunov (for the details and very interesting history of the subject, see [9]). 
Let us choose $b_{3}-b_{1}=\epsilon$ as our small parameter. Then, the equations of motion in the Kirchhoff case can be rewritten:

$$
\begin{aligned}
\dot{M}_{1} & =\left(a_{3}-a_{1}\right) M_{2} M_{3}+\epsilon\left(M_{2} p_{3}+M_{3} p_{2}\right)+\left(c_{3}-c_{1}\right) p_{2} p_{3} \\
\dot{M}_{2} & =\left(a_{1}-a_{3}\right) M_{2} M_{3}-\epsilon\left(M_{1} p_{3}+M_{3} p_{1}\right)+\left(c_{1}-c_{3}\right) p_{1} p_{3} \\
\dot{M}_{3} & =0 \\
\dot{p}_{1} & =a_{3} M_{3} p_{2}-a_{1} M_{2} p_{3}+\epsilon p_{2} p_{3} \\
\dot{p}_{2} & =a_{1} M_{1} p_{3}-a_{3} M_{3} p_{1}-\epsilon p_{1} p_{3} \\
\dot{p}_{3} & =a_{1}\left(p_{1} M_{2}-p_{2} M_{1}\right)
\end{aligned}
$$

The unperturbed system, defined by $\epsilon=0$, has a particular solution $M_{i}=$ $\frac{M_{i}^{0}}{t}, p_{i}=\frac{p_{i}^{0}}{t}$ where:

$$
\begin{array}{rlrl}
M_{1}^{0}=\frac{i \alpha}{a_{1}}, & M_{2}^{0}=\frac{i \beta}{a_{1}}, \quad & M_{3}^{0}=0, \\
p_{1}^{0}=\frac{i \beta}{\sqrt{a_{1}\left(c_{3}-c_{1}\right)}}, & p_{2}^{0}=\frac{-i \alpha}{\sqrt{a_{1}\left(c_{3}-c_{1}\right)}}, \quad p_{3}^{0}=\frac{1}{\sqrt{a_{1}\left(c_{3}-c_{1}\right)}}
\end{array}
$$

and $\alpha, \beta$ are constants that satisfy $\alpha^{2}+\beta^{2}=1$. The expressions (4), (5) are the first terms in the Laurent series for a solution $M_{i}=\frac{M_{i}^{0}}{t}+\epsilon M_{i}^{1}+\ldots$, $p_{i}=\frac{p_{i}^{0}}{t}+\epsilon p_{i}^{1}+\ldots$ of the equations (3). For the second terms one gets the following system:

$$
\begin{aligned}
\dot{M}_{1}^{1} & =\left(a_{3}-a_{1}\right) \frac{M_{2}^{0} M_{3}^{1}}{t}+\frac{M_{2}^{0} p_{3}^{0}}{t^{2}}+\left(c_{3}-c_{1}\right) \frac{p_{2}^{0} p_{3}^{1}}{t}+\left(c_{3}-c_{1}\right) \frac{p_{2}^{1} p_{3}^{0}}{t} \\
\dot{M}_{2}^{1} & =\left(a_{1}-a_{3}\right) \frac{M_{1}^{0} M_{3}^{1}}{t}-\frac{M_{1}^{0} p_{3}^{0}}{t^{2}}+\left(c_{1}-c_{3}\right) \frac{p_{1}^{0} p_{3}^{1}}{t}+\left(c_{1}-c_{3}\right) \frac{p_{1}^{1} p_{3}^{0}}{t} \\
\dot{M}_{3}^{1} & =0 \\
\dot{p}_{1}^{1} & =a_{3} \frac{M_{3}^{1} p_{2}^{0}}{t}-a_{1} \frac{M_{2}^{0} p_{3}^{1}}{t}-a_{1} \frac{M_{2}^{1} p_{3}^{0}}{t}+\frac{p_{2}^{0} p_{3}^{0}}{t^{2}} \\
\dot{p}_{2}^{1} & =-a_{3} \frac{M_{3}^{1} p_{1}^{0}}{t}+a_{1} \frac{M_{1}^{0} p_{3}^{1}}{t}+a_{1} \frac{M_{1}^{1} p_{3}^{0}}{t}-\frac{p_{1}^{0} p_{3}^{0}}{t^{2}} \\
\dot{p}_{3}^{1} & =a_{1}\left(\frac{p_{1}^{0} M_{2}^{1}}{t}+\frac{p_{1}^{1} M_{2}^{0}}{t}-\frac{p_{2}^{0} M_{1}^{1}}{t}-\frac{p_{2}^{1} M_{1}^{0}}{t}\right)
\end{aligned}
$$

First we will find the solutions of the homogeneous system

$$
\begin{array}{ll}
\dot{M}_{1}^{1}=\left(c_{3}-c_{1}\right) \frac{p_{2}^{0} p_{3}^{1}}{t}+\left(c_{3}-c_{1}\right) \frac{p_{2}^{1} p_{3}^{0}}{t}, & \dot{p}_{1}^{1}=-a_{1} \frac{M_{2}^{0} p_{3}^{1}}{t}-a_{1} \frac{M_{2}^{1} p_{3}^{0}}{t} \\
\dot{M}_{2}^{1}=\left(c_{1}-c_{3}\right) \frac{p_{1}^{0} p_{3}^{1}}{t}+\left(c_{1}-c_{3}\right) \frac{p_{1}^{1} p_{3}^{0}}{t}, & \dot{p}_{2}^{1}=a_{1} \frac{M_{1}^{0} p_{3}^{1}}{t}+a_{1} \frac{M_{1}^{1} p_{3}^{0}}{t} \\
\dot{M}_{3}^{1}=0 & \dot{p}_{3}^{1}=a_{1}\left(\frac{p_{1}^{0} M_{2}^{1}}{t}+\frac{p_{1}^{1} M_{2}^{0}}{t}-\frac{p_{2}^{0} M_{1}^{1}}{t}-\frac{p_{2}^{1} M_{1}^{0}}{t}\right)
\end{array}
$$


of the form: $M_{1}^{1}=t^{s} M, M_{2}^{1}=t^{s} N, p_{1}=t^{s} P, p_{2}=t^{s} Q, p_{3}=t^{s} R$. One gets

$$
\begin{aligned}
& s M=\left(c_{3}-c_{1}\right)\left(p_{2}^{0} R+p_{3}^{0} N\right), \quad s N=\left(c_{1}-c_{3}\right)\left(p_{1}^{0} R+p_{3}^{0} P\right) \\
& s P=-a_{1} M_{2}^{0} R-a_{1} p_{3}^{0} N, \quad s Q=a_{1} M_{1}^{0} R+a_{1} p_{3}^{0} M \\
& s R=a_{1} p_{1}^{0} N+a_{1} M_{2}^{0} P-a_{1} p_{2}^{0} M-a_{1} M_{1}^{0} Q .
\end{aligned}
$$

This is a homogeneous system of linear equations and it has nontrivial solutions only when the determinant of the system is zero:

$$
(s-1)^{3}(s+1)(s+2)=0
$$

By solving the system (잠) for $s=1, s=-1$, and $s=-2$ one gets the general solution of system (6):

$$
\begin{array}{ll}
M_{1}^{1}=\frac{i \alpha a}{t^{2}} k_{5}-\frac{a \beta}{t} k_{4}+a\left(k_{2}-i \alpha k_{3}\right) t & p_{1}^{1}=\frac{i \beta}{t^{2}} k_{5}+\frac{\alpha}{t} k_{4}+k_{1} t \\
M_{2}^{1}=\frac{i \beta a}{t^{2}} k_{5}+\frac{a \alpha}{t} k_{4}+a\left(-k_{1}-i \beta k_{3}\right) t & p_{2}^{1}=\frac{-i \alpha}{t^{2}} k_{5}+\frac{\beta}{t} k_{4}+k_{2} t \\
p_{3}^{1} & =\frac{k_{5}}{t^{2}}+k_{3} t,
\end{array}
$$

where $a=\sqrt{\frac{\left(c_{3}-c_{1}\right)}{a_{1}}}$. We will find the solution of (6) by using the standard method of variation of constants. We have:

$$
\begin{aligned}
\frac{i \alpha a}{t^{2}} k_{5}^{\prime}-\frac{a \beta}{t} k_{4}^{\prime}+a\left(k_{2}^{\prime}-i \alpha k_{3}^{\prime}\right) t & =\left(a_{3}-a_{1}\right) \frac{M_{2}^{0} M_{3}^{1}}{t}+\frac{M_{2}^{0} p_{3}^{0}}{t^{2}} \\
\frac{i \beta a}{t^{2}} k_{5}^{\prime}+\frac{a \alpha}{t} k_{4}^{\prime}+a\left(-k_{1}^{\prime}-i \beta k_{3}^{\prime}\right) t & =\left(a_{1}-a_{3}\right) \frac{M_{1}^{0} M_{3}^{1}}{t}-\frac{M_{1}^{0} p_{3}^{0}}{t^{2}} \\
\frac{i \beta}{t^{2}} k_{5}^{\prime}+\frac{\alpha}{t} k_{4}^{\prime}+k_{1}^{\prime} t & =a_{3} \frac{M_{3}^{1} p_{2}^{0}}{t}+\frac{p_{2}^{0} p_{3}^{0}}{t^{2}} \\
\frac{-i \alpha}{t^{2}} k_{5}^{\prime}+\frac{\beta}{t} k_{4}^{\prime}+k_{2}^{\prime} t & =-a_{3} \frac{M_{3}^{1} p_{1}^{0}}{t}-\frac{p_{1}^{0} p_{3}^{0}}{t^{2}} \\
\frac{k_{5}^{\prime}}{t^{2}}+k_{3}^{\prime} t & =0
\end{aligned}
$$

and one gets

$$
\begin{aligned}
& k_{1}(t)=\frac{i \alpha M_{3}^{1}}{2 a} \frac{1}{t}+k_{1}, \quad k_{2}(t)=\frac{i \beta M_{3}^{1}}{2 a} \frac{1}{t}+k_{2}, \quad k_{3}(t)=k_{3} \\
& k_{4}(t)=\frac{-i\left(2 a_{3}-a_{1}\right) M_{3}^{1}}{\sqrt{a_{1}\left(c_{3}-c_{1}\right)}} t-\frac{i}{a_{1}\left(c_{3}-c_{1}\right)} \ln (t)+k_{4}, \quad k_{5}(t)=k_{5} .
\end{aligned}
$$

Thus, $k_{4}(t)$ is not a uniform function of complex time and we get proved the following theorem:

Theorem 1. When $B \neq 0$, the Kirchhoff case of the Kirchhoff equations is not algebraic integrable system. 


\section{Three-Dimensional Chaplygin Case}

In 1897 Chaplygin (see [4]) defined a case which instead of a first integral had an invariant relation. This system has also been considered in 13 where nonintegrability of the system has been proven. The Chaplygin system is defined for $A=\operatorname{diag}\left(a_{1}, a_{2}, a_{3}\right)$ by:

$$
\begin{aligned}
& b_{13} \sqrt{a_{2}-a_{1}} \mp\left(b_{2}-b_{1}\right) \sqrt{a_{3}-a_{2}}=0, b_{12}=0 \\
& b_{13} \sqrt{a_{3}-a_{2}} \pm\left(b_{3}-b_{2}\right) \sqrt{a_{2}-a_{1}}=0, b_{23}=0 \\
& c_{13} \sqrt{a_{2}-a_{1}} \mp\left(c_{2}-c_{1}\right) \sqrt{a_{3}-a_{2}}=0, c_{12}=0 \\
& c_{13} \sqrt{a_{3}-a_{2}} \pm\left(c_{3}-c_{2}\right) \sqrt{a_{2}-a_{1}}=0, c_{23}=0
\end{aligned}
$$

The invariant relation is: $F_{4}=M_{1} \sqrt{a_{2}-a_{1}} \mp M_{3} \sqrt{a_{3}-a_{2}}=0$.

Conditions (10) may be seen as certain analogous of the Hess-Appel'rot conditions in the case of motion of a heavy rigid body fixed at a point. A geometric interpretation of Hess-Appel'rot conditions has been given by Zhukovski. Using it, one can see the Hess-Appel'rot case as a perturbation of the Lagrange top. Detailed analysis of this system as well as higher dimensional generalizations, the Lax representation and bi-Hamiltonian properties are given in [5, 7]. The class of systems of Hess-Appel'rot type is defined there also. Starting form the basic properties of this class od systems a new set of examples are constructed in [8].

Similarly, the Chaplygin case is a perturbation of the Kirchhoff case. Let us choose the basis where $a_{1}=a_{2}$. In this new basis, the Chaplygin conditions are (see for example [3]): $a_{1}=a_{2}, a_{13} \neq 0, B=\operatorname{diag}\left(b_{1}, b_{1}, b_{3}\right), C=$ $\operatorname{diag}\left(c_{1}, c_{1}, c_{3}\right)$. The Hamiltonian becomes $2 H=a_{1}\left(M_{1}^{2}+M_{2}^{2}\right)+a_{3} M_{3}+$ $2 a_{13} M_{1} M_{3}+2 b_{1}\left(M_{1} p_{1}+M_{2} p_{2}\right)+2 b_{3} M_{3} p_{3}+c_{1}\left(p_{1}^{2}+p_{2}^{2}\right)+c_{3} p_{3}^{2}$. Thus $2 H=H_{K}+2 a_{13} M_{1} M_{3}$ where $H_{K}$ is the Hamiltonian for the Kirchhoff case. In the new coordinates the invariant relation is $M_{3}=0$.

Let us apply now the method of small parameter to the Chaplygin case when $B=0$. We will consider $a_{13}$ as a small parameter $\epsilon$. The equations are:

$$
\begin{aligned}
\dot{M}_{1} & =\left(a_{3}-a_{1}\right) M_{2} M_{3}+\left(c_{3}-c_{1}\right) p_{2} p_{3}+\epsilon M_{1} M_{2} \\
\dot{M}_{2} & =\left(a_{1}-a_{3}\right) M_{2} M_{3}+\left(c_{1}-c_{3}\right) p_{1} p_{3}+\epsilon\left(M_{3}^{2}-M_{1}^{2}\right) \\
\dot{M}_{3} & =-\epsilon M_{2} M_{3} \\
\dot{p}_{1} & =a_{3} M_{3} p_{2}-a_{1} M_{2} p_{3}+\epsilon M_{1} p_{2} \\
\dot{p}_{2} & =a_{1} M_{1} p_{3}-a_{3} M_{3} p_{1}+\epsilon\left(M_{3} p_{3}-M_{1} p_{1}\right) \\
\dot{p}_{3} & =a_{1}\left(p_{1} M_{2}-p_{2} M_{1}\right)-\epsilon M_{3} p_{2}
\end{aligned}
$$

We assume $M_{3}=0$. The unperturbed system coincides with the unperturbed system of equations (3). Hence, it has a particular solution (44), (5). For the terms of order $\epsilon$, one gets the system which homogeneous part is (7). Applying again the method of variation of constants, one gets the solutions 
(9)) where

$$
\begin{aligned}
& k_{1}(t)=k_{1}, \quad k_{2}(t)=k_{2}, \quad k_{3}(t)=k_{3}, \\
& k_{4}(t)=\frac{\alpha}{a_{1} \sqrt{a_{1}\left(c_{3}-c_{1}\right)}} \ln (t)+k_{4}, \quad k_{5}(t)=k_{5} .
\end{aligned}
$$

From the Lax representation for the Clebsch case, given by Perelomov in [16, a Lax representation for the Kirchhoff case when $B=0$ can be obtained. A Lax representation for the Chaplygin case, given below is a perturbation of that one, given by Perelomov.

Theorem 2. When $B=0$, on the invariant manifold given by the invariant relation, the equations of motion of the Chaplygin case are equivalent to the matrix equation:

$$
\dot{L}(\lambda)=[L(\lambda), Q(\lambda)]
$$

where $L(\lambda)=\lambda^{2} L_{2}+\lambda L_{1}-L_{0}, Q(\lambda)=\lambda Q_{1}+Q_{0}$, and

$$
\begin{gathered}
L_{2}=\operatorname{diag}\left(c_{1} / a_{1}, c_{1} / a_{1}, c_{3} / a_{1}\right), Q_{1}=\operatorname{diag}\left(a_{1}, a_{1}, a_{3}\right) \\
L_{1}=\left[\begin{array}{ccc}
0 & -M_{3} & M_{2} \\
M_{3} & 0 & -M_{1} \\
-M_{2} & M_{1} & 0
\end{array}\right] \quad L_{0}=p p^{T} \\
Q_{0}=\left[\begin{array}{ccc}
0 & -a_{3} M_{3}-a_{13} M_{1} & a_{1} M_{2} \\
a_{3} M_{3}+a_{13} M_{1} & 0 & -a_{1} M_{1}-a_{13} M_{3} \\
-a_{1} M_{2} & a_{1} M_{1}+a_{13} M_{3} & 0
\end{array}\right]
\end{gathered}
$$

The spectral curve $\operatorname{det}(L(\lambda)-\mu \cdot 1)=0$ is:

$$
\begin{aligned}
\Gamma: \quad \mu^{3}+\mu^{2} F_{3}-\lambda_{1}^{2} \mu^{2}\left(c_{3}+2 c_{1}\right)+ \\
\\
\lambda_{1}^{2} \mu\left[2 F_{1}-\left(2 c_{1}+c_{3}\right) F_{3}\right]+\lambda_{1}^{4} \mu c_{1}\left(c_{1}+2 c_{3}\right)- \\
\quad \lambda_{1}^{6} c_{1}^{2} c_{3}-\lambda_{1}^{4}\left(2 c_{1} F_{1}-c_{1}\left(c_{1}+c_{3}\right) F_{3}\right)+\lambda_{1}^{2} a_{1} F_{2}^{2}=0 .
\end{aligned}
$$

It is singular and has an involution $\sigma:\left(\lambda_{1}, \mu\right) \rightarrow\left(-\lambda_{1}, \mu\right)$. The curve $\Gamma_{1}=\Gamma / \sigma$ is a nonsingular genus one curve.

\section{Higher-dimensional Kirchhoff equations-the Kirchhoff and Chaplygin CASES ON $e(4)$}

We will consider a generalization of the Kirchhoff equations on $e(n)$. Let us consider the Hamiltonian equations with a Hamiltonian function:

$$
2 H=\sum A_{i j k l} M_{i j} M_{k l}+2 \sum B_{i j k} M_{i j} p_{k}+\sum C_{k l} p_{k} p_{l}
$$

in the standard Lie-Poisson structure on $s o(n)$ given by:

$$
\begin{gathered}
\left\{M_{i j}, M_{k l}\right\}=\delta_{i k} M_{j l}+\delta_{j l} M_{i k}-\delta_{i l} M_{j k}-\delta_{j k} M_{i l} \\
\left\{M_{i j}, p_{k}\right\}=\delta_{i k} p_{j}-\delta_{j k} p_{i}
\end{gathered}
$$

We will restrict ourselves to dimension four. A four-dimensional Kirchhoff case should have two linear first integrals: $M_{12}$ and $M_{34}$. It is interesting that under such assumption, the "mixed" term in the Hamiltonian is missing. 
Proposition 1. If $M_{12}$ and $M_{34}$ are the first integrals, then $B_{i j k}=0$.

Proof. Proof is based on the facts that $\dot{M}_{12}=\left\{M_{12}, H\right\}, \dot{M}_{34}=\left\{M_{34}, H\right\}$ and follows by a direct calculations.

Definition 1. The four-dimensional Kirchhoff case is defined by

$$
\begin{aligned}
2 H_{K}= & A_{1212} M_{12}^{2}+A_{1313}\left(M_{13}^{2}+M_{14}^{2}+M_{23}^{2}+M_{24}^{2}\right)+A_{3434} M_{34}^{2}+ \\
& A_{1234} M_{12} M_{34}+C_{11}\left(p_{1}^{2}+p_{2}^{2}\right)+C_{33}\left(p_{3}^{2}+p_{4}^{2}\right)
\end{aligned}
$$

Theorem 3. The four dimensional Kirchhoff case is completely integrable in Liouville sense.

Proof. On e(4) the standard Lie - Poisson structure has two Casimir functions:

$$
\begin{aligned}
F_{1}= & p_{1}^{2}+p_{2}^{2}+p_{3}^{2}+p_{4}^{2}, \\
F_{2}= & \left(M_{13} p_{4}-M_{14} p_{3}+M_{34} p_{1}\right)^{2}+\left(M_{23} p_{1}+M_{12} p_{3}-M_{13} p_{2}\right)^{2}+ \\
& \left(M_{24} p_{1}-M_{14} p_{2}+M_{12} p_{4}\right)^{2}+\left(M_{23} p_{4}+M_{34} p_{2}-M_{24} p_{3}\right)^{2}
\end{aligned}
$$

Thus, general symplectic leaves are 8-dimensional and for complete integrability one needs four first integrals in involution. Except Hamiltonian, we have two linear first integrals $F_{3}=M_{12}, F_{4}=M_{34}$ and one additional quadratic first integral:

$$
\begin{aligned}
F_{5} & =a_{1}\left(M_{12} M_{34}+M_{14} M_{23}-M_{13} M_{24}\right)^{2} \\
& -c_{1}\left(\left(M_{13} p_{4}-M_{14} p_{3}+M_{34} p_{1}\right)^{2}+\left(M_{23} p_{4}+M_{34} p_{2}-M_{24} p_{3}\right)^{2}\right) \\
& -c_{3}\left(\left(M_{23} p_{1}+M_{12} p_{3}-M_{13} p_{2}\right)^{2}+\left(M_{24} p_{1}-M_{14} p_{2}+M_{12} p_{4}\right)^{2}\right)
\end{aligned}
$$

In the case of four-dimensional Chaplygin case, one can naturally assume that $M_{12}$ and $M_{34}$ are invariant relations. From this assumption, we get:

Definition 2. The four-dimensional Chaplygin case of the Kirchhoff equations on e(4) is defined by the Hamiltonian:

$$
\begin{aligned}
2 H_{C h}= & A_{1212} M_{12}^{2}+A_{1313}\left(M_{13}^{2}+M_{14}^{2}+M_{23}^{2}+M_{24}^{2}\right)+A_{3434} M_{34}^{2}+ \\
& A_{1234} M_{12} M_{34}+A_{1213} M_{12} M_{13}+A_{1214} M_{12} M_{14}+ \\
& A_{1223} M_{12} M_{23}+A_{1224} M_{12} M_{24}+A_{1334} M_{13} M_{34}+ \\
& A_{1434} M_{14} M_{34}+A_{2334} M_{23} M_{34}+A_{2434} M_{24} M_{34}+ \\
& B_{121} M_{12} p_{1}+B_{122} M_{12} p_{2}+B_{123} M_{12} p_{3}+B_{124} M_{12} p_{4}+ \\
& B_{341} M_{34} p_{1}+B_{342} M_{34} p_{2}+B_{343} M_{34} p_{3}+B_{344} M_{34} p_{4}+ \\
& C_{11}\left(p_{1}^{2}+p_{2}^{2}\right)+C_{33}\left(p_{3}^{2}+p_{4}^{2}\right) .
\end{aligned}
$$

One can easily check that in this case $M_{12}$ and $M_{34}$ are really the invariant relations. 


\section{ACKNOWLEDGMENTS}

The research was partially supported by the Serbian Ministry of Education and Science, Project 174020 Geometry and Topology of Manifolds, Classical Mechanics and Integrable Dynamical Systems and by the Mathematical Physics Group of the University of Lisbon, Project Probabilistic approach to finite and infinite dimensional dynamical systems, PTDC/MAT/104173/2008. The authors would like to express their gratitude to Academician V. V. Kozlov for fruitful discussions and to Professor A. V. Borisov for helpful remarks.

\section{REFERENCES}

[1] M. Adler, P. van Moerbeke: The complex geometry of the Kowalewski-Painlevé analysis, Invent. Math., 97, (1989), 3-51.

[2] M. Adler, P. van Moerbeke, P. Vanhaecke: Algebraic integrability, Painlevé geometry and Lie algebras, A Series of Modern Surveys in Mathematics, 47, Springer-Verlag, Berlin, (2004).

[3] A. V. Borisov, I. S. Mamaev: Rigid body dynamics, R\&C Dynamics, Moscow-Izhevsk, 2001, [in Russian]

[4] S. A. Chaplygin: Selected works, Nauka, Moscow, (1976), [in Russian]

[5] V. Dragović, B. Gajić: An L-A pair for the Hess-Apel'rot system and a new integrable case for the Euler-Poisson equations on $\mathrm{so}(4) \times \mathrm{so}(4)$, Roy. Soc. of Edinburgh: Proc A, 131 (2001), 845-855.

[6] V. Dragović, B. Gajić: The Lagrange bitop on so(4) $\times$ so(4) and geometry of Prym varieties, American Journal of Mathematics, 126, (2004), 981-1004.

[7] V. Dragović, B. Gajić: Systems of Hess-Appel'rot type, Comm. Math. Phys, 265, (2006), 397-435.

[8] V. Dragović, B. Gajić, B. Jovanović: Systems of Hess-Appel'rot type and Zhukovskii property, Inter. Journal of Geom. Meth in Mod. Phys, 6, No 8, (2009), 1253-1304.

[9] V. V. Golubev: Lectures on integration of the equations of motion of a rigid body about a fixed point, Moskva, Gostenhizdat, (1953) [in Russian]; English translation: Transl. Philadelphia, PA: Coronet Books, (1953).

[10] G. R. Kirchhoff: Vorlesungen über Mathematische Physics. Mechanik, Leipzig, (1874)

[11] S. Kowalevski: Sur le problème de la rotation d'un corps solide autour d'un point fixe, Acta Math. 12 (1889), 177-232.

[12] V. V. Kozlov: Methods of qualitative analysis in the dynamics of a rigid body, MGU, Moscow, (1980) [in Russian].

[13] V. V. Kozlov, D. A. Onischenko: Nonintegrability of Kirchhoff equations, Doklady ANSSSR, 266, No. 6, (1982), 1298-1300.

[14] H. Lamb: Hydrodynamics, N.Y. Dover publ., (1945).

[15] A. Lesfari: Prym varieties and applications, J. Geom. Phys., 58, (2008), 1063-1079.

[16] A. M. Perelomov: Some remarks on the integrability of the equations of motion of a rigid body in an ideal fluid, Funkc. Anal. and its Appl., 15, No.2, (1981), 83-85

${ }^{1}$ Mathematical Institute, Serbian Academy of Science and Art, Kneza Mihaila 36, 11000 Belgrade, Serbia

2 GFM, University of Lisbon, Portugal

E-mail address: vladad@mi.sanu.ac.rs and gajab@mi.sanu.ac.rs 